\title{
High Precision Antenna Characterisation for Broadband Synthetic Aperture Radar Processing
}

\author{
Marc Jäger, Bernd Gabler, Andreas Reigber \\ Microwaves and Radar Institute, Department of SAR Technology, German Aerospace Center (DLR)
}

\begin{abstract}
The airborne F-SAR sensor[1], developed and operated by the DLR-HR institute, acquires very high resolution, fully polarimetric synthetic aperture radar (SAR) data in multiple frequency bands in addition to providing a number of interferometric imaging modes. The high resolution/high bandwidth regime of operation introduces significant challenges in terms of data calibration as well as image formation, or focusing. This paper reviews the difficulties encountered in both areas and highlights the importance of compensating for the characteristics of the antenna in overcoming them. A prerequisite to doing so are highly accurate measurements of these characteristics, which are themselves difficult due to the fact that they must accurately portray interactions with the surroundings when mounted on the aircraft. The results presented concern the antenna characterisation itself and illustrate how the quality of real polarimetric and interferometric SAR data can be significantly improved when these characteristics are properly taken into account.
\end{abstract}

Index Terms-SAR Processing, Compact Test Range, Antenna Characterisation.

\section{INTRODUCTION}

SAR sensors operate by repeatedly illuminating targets with high bandwidth chirp signals as the sensor platform moves. The raw data thus obtained is defocused, due to the length of the emitted pulse and the width of the antenna beam in flight direction. SAR processing consists of refocusing targets on the basis of precise knowledge of the transmitted pulse and the sensor motion.

To obtain high quality, well calibrated SAR measurements, the processing chain must take into account the entire signal pathway through the instrument, i.e. the way in which signals in transit are affected by the impulse response of all components in the system. As SAR sensors are inherently coherent, this includes the impact on amplitude as well as phase.

Whereas most components in the sensor have one dimensional transfer functions, the antenna that transmits and receives radar signals is anisotropic as a function of direction in space and therefore requires a three dimensional characterisation (two angles and frequency). Compensating for the antenna is therefore a particularly challenging step in SAR processing and also requires a precise, three dimensional characterisation of the antenna as an input.

The F-SAR sensor, shown in figure 1, carries its antennas in a specially designed carrier on the right side of the fuselage. Signals transmitted and received by the antennas interact with the structure of the antenna carrier to some degree depending on the frequency band and antenna position. The antenna characterisation must faithfully reproduce these interactions

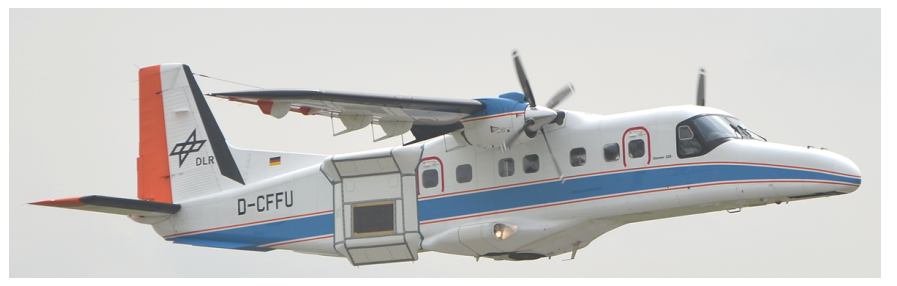

Fig. 1. The F-SAR sensor installed in a DO-228 aircraft with the antenna carrier, carrying X,C,S and L-band antennas, visible toward the aft.

in order to accurately describe effects encountered the data acquired. As explained in section II, this significantly complicates the measurement process.

The antenna diagrams thus obtained are an essential input to the processing chain and impact all aspects of sensor calibration. The so-called radiometric calibration, which compensates for systematic variations in image intensity, must take into account the spatially anisotropic antenna gain in combination with the sensor attitude angles to arrive at an appropriate correction at each point in a given acquisition. The importance of using a full $2 \mathrm{D}$ description of the antenna gain to do so is discussed in section III.

In SAR polarimetry, acquisitions comprise several channels acquired simultaneously in different polarisations. In terms of calibration, it is of importance that these channels be well balanced. In addition to the radiometry, this also includes the inter-channel phase differences, and the resulting role of accurate antenna phase measurements in the calibration process is discussed in section IV.

As described in section $\mathrm{V}$, phase also plays a central role in SAR interferometry. The results presented concern the compensation of artefacts observed due to interactions between the antennas of the F-SAR X-Band interferometer and the structure of the antenna carrier.

Finally, as described in section VI, the frequency response of the antenna can have an adverse effect on the image formation itself. The resulting inability to properly focus SAR data is a problem that is especially noticeable for high bandwidth systems such as F-SAR, where it becomes infeasible to design and build antennas with an ideal frequency response and the frequency response itself may even vary as a function of propagation direction. 


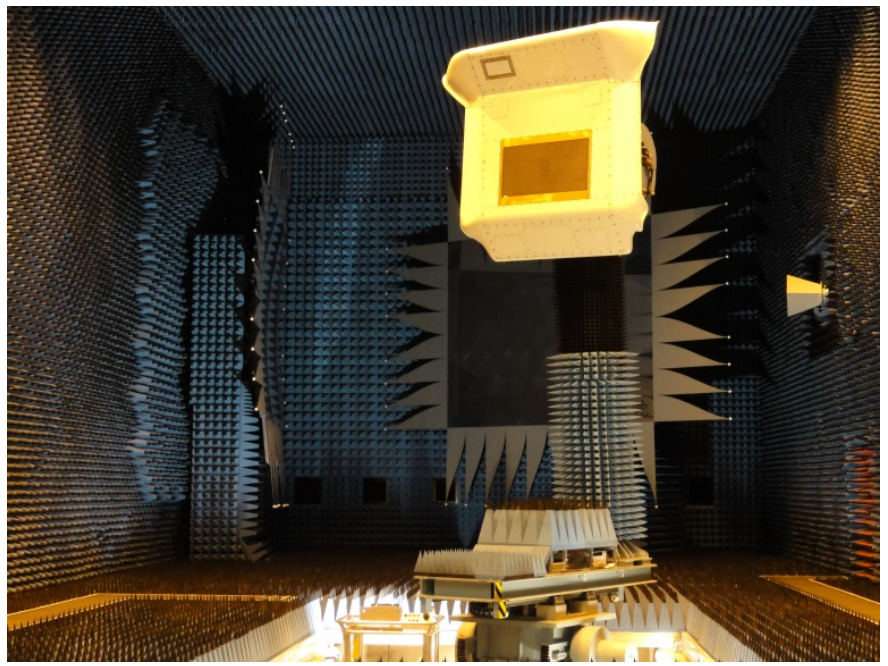

Fig. 2. The F-SAR antenna carrier in the compact test range.

\section{ANTENNA CHARACTERISATION}

Reliable radiation pattern simulation of imaging radar antennas for airborne platforms still exceeds the capabilities of todays development tools. Especially with arrangements of several elevation beam steering antennas for polarimetric SAR interferometry, the ambient aircraft cabin shows significant influence on the amplitude and phase characteristics of each individual radiation pattern. Therefore, the complete F-SAR antenna carrier structure is installed in the DLR Compact Test Range [6] to determine the antenna parameters in a complete hemisphere.

Installed on a 6-axis positioner that is capable to carry AUTs with up to $300 \mathrm{~kg}$, 3D theta-phi antenna patters are obtained in a roll over azimuth acquisition[2][3].

\section{A. Measurement Procedure}

Antennas at different offset positions to the rotational center of the measurement axis and with their individual propagation delay with respect to the system phase reference can be characterized precisely in both magnitude and phase, when signal theory constraints are carefully taken into account. Nyquists theorem for the maximum increment has to be fulfilled for the scan (azimuth) and for the step (roll) axis of the AUT positioner:

$$
\Delta_{\max }=\frac{1}{2} \operatorname{atan}\left(\frac{c}{(R+r) f_{\max }}\right),
$$

where $\Delta_{\max }$ denotes the maximum angular increment of the positioner, $c$ is the speed of light, $R$ is the distance from the rotation center to the antenna phase center, $r$ is the radius of the antenna and $f_{\max }$ denotes the maximum frequency.

Capturing a fully-polarimetric hemisphere of a typical airborne X-Band SAR antenna with the two simultaneous measurement channels in the Compact Test Range requires only one hour for the bare antenna. The measurement time extends
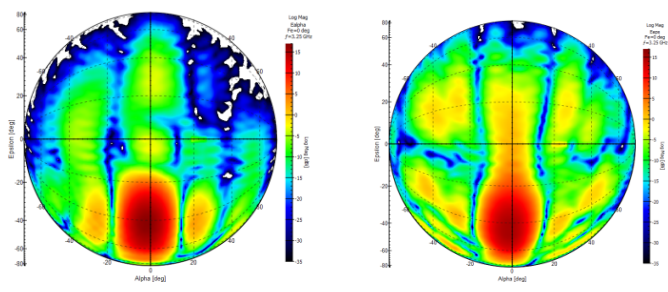

Fig. 3. F-SAR S-band antennas on the top plate of the antenna carrier, magnitude plots, left $\mathrm{HH}$, right VV.
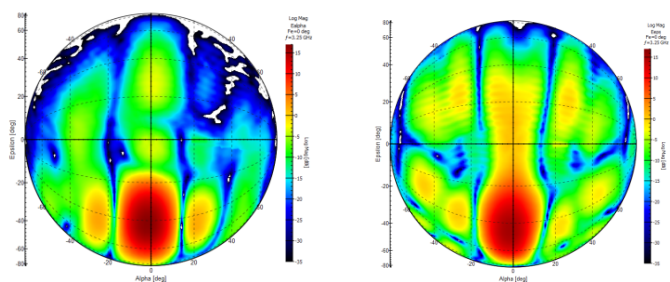

Fig. 4. F-SAR S-band antennas on the bottom plate of the antenna carrier, magnitude plots, left $\mathrm{HH}$, right $\mathrm{VV}$

to 36 hours for the same antenna on the carrier structure due to the smaller increments in both the scan and the step axis. Motion parameters of the positioner and the measurement time of the analyzer are monitored for each individual sweep and are made available in the measurement processing chain. With increasing acquisition time, these corrections are essential for the phase information of the time consuming higher radar band measurements.

A large sweep bandwidth of the network analyzer signals allows an adequate downrange resolution that facilitates time domain post-processing of the acquired antenna data. Positioner velocity must allow for a high number of frequency increments within the sweep time of the signal analyzer to avoid unwanted range aliasing effects. With this method, the investigated radiating surface is enlarged from the bare antenna to the complete antenna carrier structure. The electromagnetic vectors of all parasitic scatters are superimposed with that of the radiating antenna element. Measurements now include multi-path effects on the structure and scattering on edges that influence the antenna patterns in amplitude and phase.

\section{B. Benefit of this Method}

The electrical distance of the antennas phase center in three dimensional space, relative to the rotational origin of the measurement system, can be precisely estimated from the phase history of the acquired antenna data. Measurement results show that antennas have individual phase centers for $\mathrm{H}-$ and V-polarization that may be in front or behind the surface of the aperture. Moreover, there is a significant influence of the carrier structure, depending on the antenna position and the polarization.

As explained in further detail in section IV, these offsets are important in the calibration of polarimetric SAR data. 

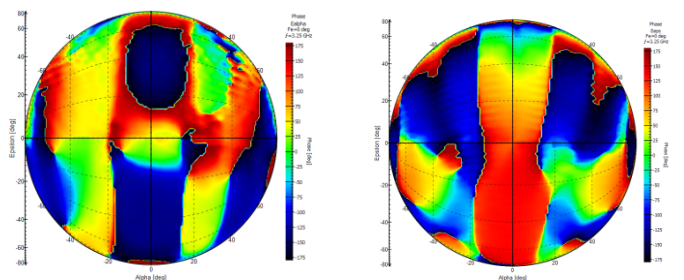

Fig. 5. F-SAR S-band antennas on the bottom plate of the antenna carrier phase plots after offset correction, left $\mathrm{HH}$, right VV
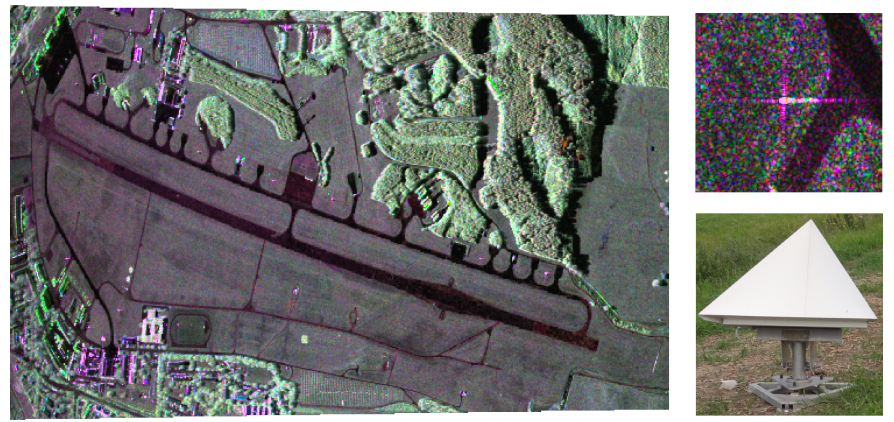

Fig. 6. Left: Part of a polarimetric data set acquired by the F-SAR sensor at $\mathrm{X}$-Band. The red green and blue channels correspond to the HH, HV and VV polarisations, respectively. Right: A trihedral corner reflector used to verify image quality as seen by the SAR sensor (top) and in the field (bottom).

\section{RADIOMETRIC CALIBRATION}

The aim of radiometric calibration, as reviewed in [7], is to link the intensity in the focused SAR image to the radar cross section (RCS) of the targets it contains. Among other things, this implies that the processor must remove systematic variations in image brightness that arise due to the anisotropy of the antenna gain in combination with variations in the aircraft attitude.

As explained in section II,high resolution, complex 3D antenna patterns in elevation $\theta_{e l}$ squint $\theta_{s q}$ and frequency $f$ are available for all transmit and receive polarisations. The 2D antenna gain, for a given transmit and receive combination $\{T, R\}$, is given by integrating over the processed frequency range:

$$
G_{A}\left(\theta_{e l}, \theta_{s q}\right)=\sum_{f \in\left\{f_{0}, f_{1}\right\}} W_{R}(f)^{2} \prod_{c \in\{R, T\}}\left|g_{c}\left(\theta_{e l}, \theta_{s q}, f\right)\right|^{2},
$$

where $f_{0}$ and $f_{1}$ denote the start and end frequency of the transmitted chirp, $W_{R}(f)$ is an apodisation function applied for sidelobe suppression by the processor and $g_{\{T, R\}}$ are the measured one-way complex antenna patterns.

Roughly speaking, the calibration process, the technical details of which are described in [4], revolves around computing how much energy reaches each resolution cell of a SAR acquisition and compensating for any imbalances within the scene. This is achieved by integrating the energy transmitted towards each resolution cell over the illumination time, taking into account the antenna pattern $G_{A}$ and the recorded sensor
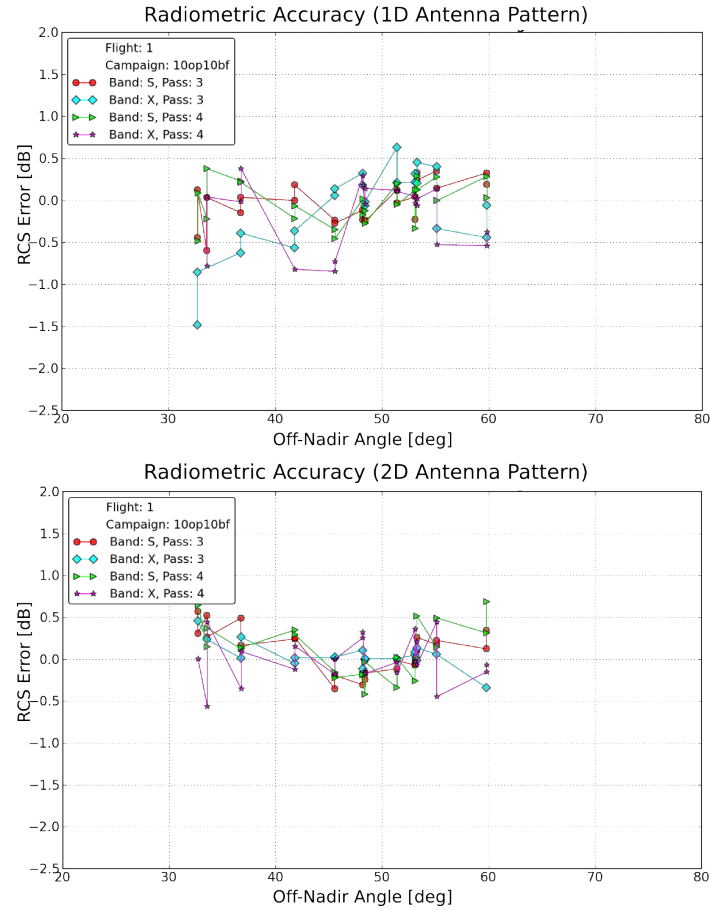

Fig. 7. A comparison of theoretical and measured radar cross sections for trihedral reflectors in the F-SAR calibration test site in Kaufbeuren, Germany. Top: Using a one dimensional elevation pattern measured at the centre frequency. Bottom: Using a two dimensional antenna pattern integrated over the sensor bandwidth. The plots are based on four independent acquisitions in $\mathrm{X}$ and $\mathrm{S}$ Band, acquired with a bandwidth of $300 \mathrm{MHz}$.

attitude angles.

To illustrate the importance of using the full two dimensional antenna pattern and taking into account the entire sensor bandwidth, figure 7 compares two approaches to calibration. Each point in plots represents a trihedral reflector while each line corresponds to a single acquisition. The first uses the one dimensional elevation diagram measured at the centre frequency of the sensor. The second uses the two dimensional diagram integrated over the acquisition bandwidth of 300 $\mathrm{MHz}$. The simpler approach clearly produces more noisy results, due to the fact that sensor attitude angles cannot be compensated adequately, and introduces a slight trend as a function of elevation, because the sensor bandwidth has not been accounted for. Taken together, the approach leveraging the full three dimensional antenna characterisation improves the observed RCS standard deviation from $0.36 d B$ to $0.26 d B$.

\section{Polarimetric CAlibration}

Polarimetric SAR acquisitions comprise multiple channels with different transmit and receive polarisations acquired simultaneously. In addition to radiometric accuracy, polarimetric calibration also concerns the inter-channel phase differences. To improve the phase calibration, the phase information in the 3D antenna pattern is exploited in a fashion similar to the one adopted for radiometric calibration.

Initially, a phase map is extracted from the $3 \mathrm{D}$ antenna 


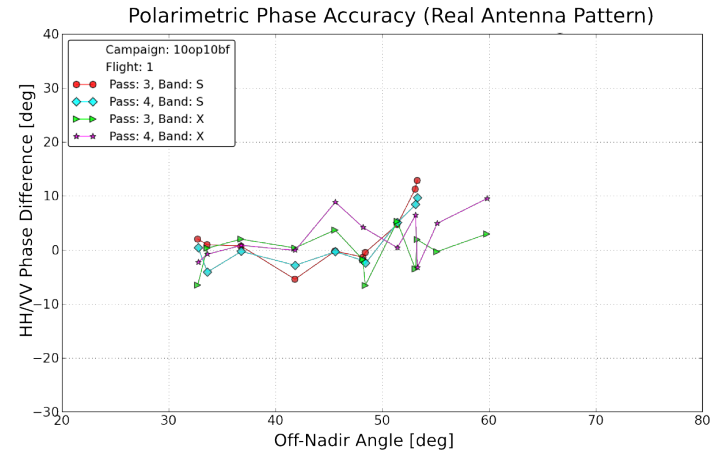

Polarimetric Phase Accuracy (Complex Antenna Pattern)

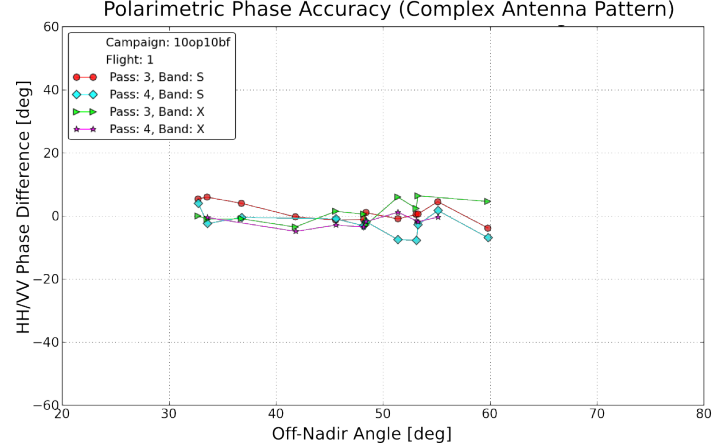

Fig. 8. The phase difference between the HH and VV polarised channels measured on trihedral corner reflectors. The difference is expected to be zero. Top: Observed differences without the antenna phase correction. Bottom: Phase differences after the correction.

pattern at the sensor's center frequency $f_{c}$ :

$$
P_{A}\left(\theta_{e l}, \theta_{s q}\right)=\prod_{c \in\{T, R\}} \frac{g_{c}\left(\theta_{e l}, \theta_{s q}, f_{c}\right)}{\left|g_{c}\left(\theta_{e l}, \theta_{s q}, f_{c}\right)\right|}
$$

A phase correction $\Delta_{\phi}$ for a given resolution cell is then obtained by integrating, for each resolution cell in an acquisition and taking into account sensor attitude angles, the the antenna phase contribution over the illumination time. A more detailed description is given in [5].

Figure 8 illustrates the improvement of polarimetric calibration quality attained by introducing an antenna phase correction during processing. The phase standard deviation as measured on trihedral calibration targets has decreased from 4.6 to 3.4 degrees.

A further important contribution of the antenna measurement are the exact positions of the antenna phase centres. As stated in section II, the $\mathrm{H}$ and $\mathrm{V}$ polarised antennas are physically located at the same point in space but their phase centres are not. Failing to take this slight offset into account would introduce systematic phase trends and the fact that these are not observed here indicates that the phase centres have been accurately located.

\section{INTERFEROMETRY}

Much the same effects are seen in SAR interferometry, where the phase difference between two independent SAR acquisitions is measured to, for example, derive digital elevation models. If the two acquisitions are acquired with different antennas, as in the example of figure 9, the differential antenna

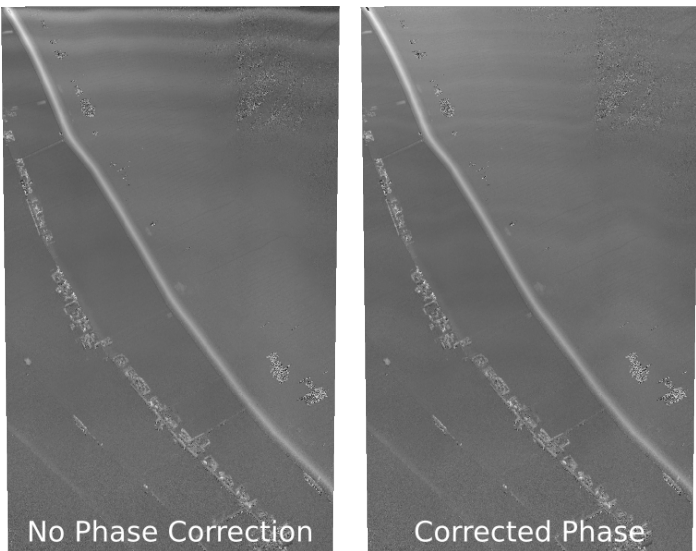

Fig. 9. The interferometric phase measured by the F-SAR single pass across track interferometer at X-Band. Left: Without antenna phase correction with visible artefacts in the top half of the measurement. Right: After the correction.

phase becomes superimposed on the actual interferometric phase measurement. In the example shown, the antenna phase correction during processing largely suppresses the artefacts that otherwise arise due to interactions between the antennas and the carrier.

\section{IMAGE FORMATION}

In addition to playing a central role in calibration, the signal propagation characteristics in the antenna can also have an effect on the image quality, i.e. the ability of the processor to accurately focus the raw data.

Especially when the relative bandwidth $\left(f_{1}-f_{0}\right) / f_{c}$ is large, the antenna can induce a noticeable weighting on the spectrum of the transmitted chirp. This weighting adversely affects impulse response and resolution after range compression. Moreover, this weighting can, in general, vary as a function of propagation direction.

Considering only the amplitude of the antenna's frequency response over elevation at beam center, the induced weighting is given by removing the overall gain from the measured twoway antenna pattern to give

$$
R_{A}\left(\theta_{e l}, f\right)=\sqrt{\frac{\sum_{f \in\left\{f_{0}, f_{1}\right\}} W_{R}(f)^{2}}{G_{A}\left(\theta_{e l}, 0\right)}} \prod_{c \in\{T, R\}}\left|g_{c}\left(\theta_{e l}, 0, f\right)\right| .
$$

If this weighting is constant over elevation, the corresponding correction can be applied directly to the known transmit chirp before range focusing. Alternatively, the correction can be carried out adaptively via short-time Fourier transforms before focusing. Figure 10 shows results for the latter type of adaptive correction at $\mathrm{S}$-band; the correction is seen to remove, to a large extent, the range trend in the range resolution evident in the upper curve. The final resolution is close to the nominal resolution of $0.64 \mathrm{~m}$, corresponding to the $300 \mathrm{MHz}$ system bandwidth, over a wide range of off-nadir angles. 


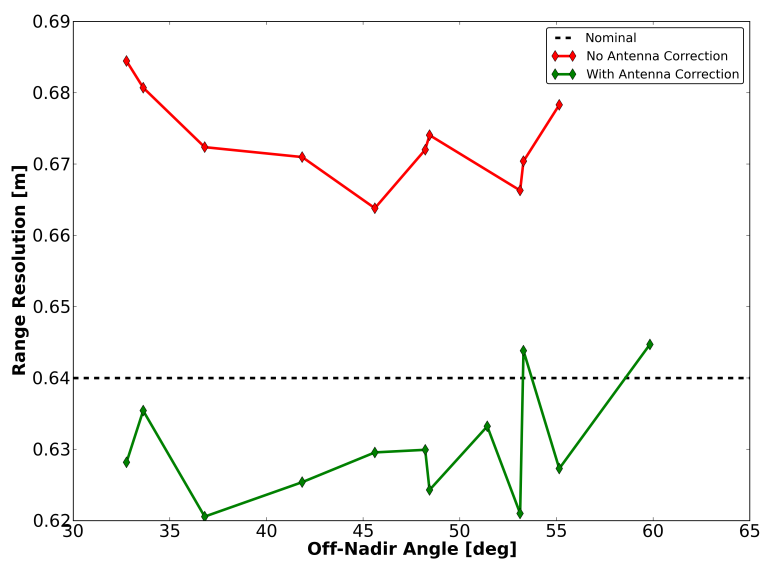

Fig. 10. A comparison of range resolution at S-Band, HH polarisation, with and without the range adaptive antenna response correction.

\section{CONCLUSION}

The results presented underline the importance of highly accurate, complex 3D antenna characterisation in the processing and calibration of broadband SAR data. Experiments using real SAR data have confirmed that compensating for propagation effects in the antenna affects all areas of SAR image quality: the radiometric calibration, the calibration of inter-channel phase differences in SAR polarimetry and, finally, the quality of interferometric phase measurements. Even the image resolution was found to be impacted by the, potentially directionally variant, frequency response of the antenna. This latter effect will become increasingly relevant in future high resolution SAR systems, where it will be increasingly infeasible to design antennas with an ideal response over the ever greater bandwiths considered.

\section{REFERENCES}

[1] Reigber, A.; Jäger, M.; Fischer, J.; Horn, R.; Scheiber, R.; Prats, P.; and Nottensteiner, A.: System Status and Calibration of the F-SAR Airborne SAR instrument. Proc. IEEE. IGARSS 2011, Jul. 2011, Vancouver, Canada

[2] A.C. Ludwig: The Definition of Cross Polarization. IEEE Trans. on Antennas and Propagation, Vol. AP-21 no 1, Jan. 1973, pp. 116-119.

[3] J. E. Roy and L. Shafai: Generalization of the Ludwig-3 Definition for Linear Copolarization and Cross Polarization IEEE Trans. on Antennas and Propagation, Vol.. 49, No. 6, June 2001, pp. 1006-1020.

[4] Reigber, A.; Scheiber, R.; Jäger, M.; Prats, P.; Hajnsek, I.; Jagdhuber, T. Papathanassiou, K.; Nannini, M.; Aguilera, E.; Baumgartner, S; Horn, R.; Nottensteiner, A. and Moreira, A.: Very-High-Resolution Airborne Synthetic Aperture Radar Imaging: Signal Processing and Applications, Proceedings of the IEEE, 101 (3), pp. 759-783. IEEE. ISSN 0018-9219. Mar. 2013.

[5] Jäger, M.; Reigber, A. and Scheiber, R.; Accurate consideration of Sensor Parameters in the Calibration and Focusing of F-SAR Data. In: Proceedings of the European Conference on Synthetic Aperture Radar (EUSAR), pp. 20-23. VDE Verlag GmbH. European Conference on Synthetic Aperture Radar (EUSAR), 2012-04-23 - 2010-04-26, Aachen, Germany.

[6] Limbach, M.; Gabler, B.; Horn, R. and Reigber, A.: DLR-HR Compact Test Range Facility, Proc. European Conference on Antennas and Propagation (EuCAP), pp. 1-4.,Mar. 2009, Berlin, Germany
[7] Freeman, A.: SAR Calibration: a Review, IEEE Trans. Geosci. Remote Sensing, vol. 30, pp. 1107-1121, Nov. 1992. 